\title{
Graph products and new solutions to Oberwolfach problems
}

\author{
Gloria Rinaldi \\ Dipartimento di Scienze e Metodi dell'Ingegneria \\ Università di Modena e Reggio Emilia \\ 42100 Reggio Emilia, Italy \\ gloria.rinaldi@unimore.it
}

\author{
Tommaso Traetta \\ Dipartimento di Matematica \\ Università Sapienza di Roma \\ 00185 Roma, Italy \\ traetta@mat.uniroma1.it
}

Submitted: Sep 21, 2010; Accepted: Feb 17, 2011; Published: Mar 11, 2011

Mathematics Subject Classifications: 05C51, 05C70, 05C76

\begin{abstract}
We introduce the circle product, a method to construct simple graphs starting from known ones. The circle product can be applied in many different situations and when applied to regular graphs and to their decompositions, a new regular graph is obtained together with a new decomposition. In this paper we show how it can be used to construct infinitely many new solutions to the Oberwolfach problem, in both the classic and the equipartite case.
\end{abstract}

\section{Introduction}

In this paper we will only deal with undirected simple graphs. For each graph $\Gamma$ we will denote by $V(\Gamma)$ and $E(\Gamma)$ its vertex-set and edge-set, respectively. By $K_{v}$ we will denote the complete graph on $v$ vertices and by $K_{\{s: r\}}$ the complete equipartite graph having $r$ parts of size s.

The number of edges incident with a vertex $a$ is called the degree of $a$ in $\Gamma$ and is denoted $d_{\Gamma}(a)$. We will drop the index referring to the underlying graph if the reference is clear. All over the paper we will consider graphs without isolated vertices, i.e., vertices of degree zero. It is well known that a graph in which all vertices have the same degree $t$ is called t-regular or simply regular.

By $C_{n}=\left(a_{1}, \ldots, a_{n}\right)$ we will denote a cycle of length $n$, namely a simple graph with vertices $a_{1}, \ldots, a_{n}$ and edges $\left[a_{i}, a_{i+1}\right]$, where the indices are to be considered modulo $n$. Also, by $\Gamma_{1} \sqcup \Gamma_{2}$ we will denote the disjoint union of two graphs, namely $V\left(\Gamma_{1}\right) \cap V\left(\Gamma_{2}\right)=\emptyset$, $V\left(\Gamma_{1} \sqcup \Gamma_{2}\right)=V\left(\Gamma_{1}\right) \cup V\left(\Gamma_{2}\right)$ and $E\left(\Gamma_{1} \sqcup \Gamma_{2}\right)=E\left(\Gamma_{1}\right) \cup E\left(\Gamma_{2}\right)$.

A decomposition of a graph $K$ is a set $\mathcal{F}=\left\{\Gamma_{1}, \ldots, \Gamma_{t}\right\}$ of subgraphs of $K$ whose edges partition, altogether, the edge-set of $K$. If all graphs $\Gamma_{i}$ are isomorphic to a given graph 
$\Gamma$, such a decomposition is generally called a $\Gamma$-decomposition of $K$. If $k$ is a positive integer, a $k$-factor of a graph $K$ is a $k$-regular spanning subgraph and a $k$-factorization of $K$ is a decomposition of $K$ into $k$-factors.

The problem of determining whether a given graph $K$ admits a $\Gamma$-decomposition, for a specified graph $\Gamma$, or admits a $k$-factorization with specified properties (for example, on the type of factors or on the automorphism group) can be very difficult to solve. A wide literature exists on these topics, too wide to be mentioned here; therefore, we refer the reader to [14].

Considerable attention has been devoted to the so called Oberwolfach problem, both in its classic and generalized formulations.

When $v$ is an odd integer, the classic Oberwolfach problem $O P(F)$ asks for a 2-factorization of the complete graph $K_{v}$ in which any 2-factor is isomorphic to the 2-factor $F$. This problem was posed by Ringel and first mentioned in [19]. In [21] the authors consider a variant of the Oberwolfach problem asking for a 2-factorization of $K_{v}-I$, the complete graph on an even set of $v$ vertices minus a 1 -factor $I$, into isomorphic 2 -factors, and the same notation $O P(F)$ is used. Obviously, in both cases, the 2-factor $F$ is a disjoint union of cycles. The notation $F\left(l_{1}^{s_{1}}, \ldots, l_{r}^{s_{r}}\right)$ will be used to denote a 2-factor consisting of $s_{i}$ cycles of length $l_{i}$ for $i=1, \ldots, r\left(s_{i}\right.$ omitted when equal to 1) and $O P\left(l_{1}^{s_{1}}, \ldots, l_{r}^{s_{r}}\right)$ will denote the corresponding Oberwolfach problem. With the same meaning, if $L_{1}, \ldots, L_{h}$ are multisets of integers we will set $F\left(L_{1}^{s_{1}}, \ldots, L_{h}^{s_{h}}\right)$ and $O P\left(L_{1}^{s_{1}}, \ldots, L_{h}^{s_{h}}\right)$. The notation $L_{i}^{s_{i}}$ means that all integers in $L_{i}$ are repeated $s_{i}$ times. With the notation $t L_{i}$ the integers in the multiset $L_{i}$ have to be multiplied by $t$. We refer to [5] for a survey on known results. In particular, it is well known that $O P(4,5)$, $O P(3,3,5), O P(3,3)$ and $O P(3,3,3,3)$ have no solutions and up to now there is no other known instance with no solution. The Oberwolfach problem $O P(m, m, \ldots, m), m \geq 3$, was completely solved in [1] in the classic case and in [20] for $v$ even. The special case $m=3$, the famous Kirkman's schoolgirl problem, was solved in [28]. Moreover every instance (except for those mentioned above) has a solution when $v \leq 40$ [15], together with a large number of other special cases for which we refer to [5]. Nevertheless, as $v$ increases, the known results solve only a small fraction of the problem and a general answer seems really hard to find. Recently, complete solutions to the Oberwolfach problem for an infinite set of orders were found in [6]. Moreover it is proved in [4] that when $v$ is even, $O P(F)$ has a solution for any bipartite 2-factor $F$. In [22] the author gave a generalization of the problem considering 2 -factorizations of the complete equipartite graph $K_{\{s: r\}}$ into isomorphic 2-factors. Obviously this generalization reduces to the classic Oberwolfach problem when $s=1$ and to the variant of [21] when $s=2$. We will use the same notations as before, namely $O P\left(s: r ; l_{1}^{t_{1}}, \ldots, l_{h}^{t_{h}}\right)$ will denote the Oberwolfach problem for the complete equipartite graph $K_{\{s: r\}}$ in which all 2-factors are of type $\left(l_{1}^{t_{1}}, \ldots, l_{h}^{t_{h}}\right)$. Moreover, in [23], the problem was completely solved in case the 2-factors are uniform of length $t$, i.e., all cycles have the same length $t, t \geq 3$. The generalized Oberwolfach problem is denoted by $O P(s: r ; t)$ in this case and it is proved in [23] that it has a solution if and only if $r s$ is divisible by $t, s(r-1)$ is even, $t$ is even if $r=2$ and $(r, s, t) \neq(3,2,3),(3,6,3),(6,2,3),(2,6,6)$. This result reduces to that found 
by Piotrowski in [27] when the complete bipartite graph is considered. Moreover, the complete bipartite graph $K_{\{s: 2\}}$ does not contain cycles of odd length; hence, its 2-factors can only have cycles of even length. Also, it admits a 2 -factorization only if $s$ is even. In [27], Piotrowski proved the sufficiency of all these conditions when $s \neq 6$, namely he proved that $O P\left(s: 2 ; 2 c_{1}, \ldots, 2 c_{t}\right)$ has a solution for each set $\left\{c_{1}, \ldots, c_{t}\right\}$ with $\sum c_{i}=s$ and $c_{i} \geq 2$, except for $O P(6: 2 ; 6,6)$ which has no solution. This completely solves the problem for the bipartite case.

When speaking of a symmetric solution $\mathcal{F}$ to the Oberwolfach problem we mean that $\mathcal{F}$ admits an automorphism group $G$ whose action on a set of objects (mainly vertices, edges or factors) satisfies some properties. A classification result has been achieved in the case where $G$ acts 2 -transitively on the set of vertices, [3]. The case where $G$ acts sharply transitively on the vertex-set has been considered in [9]. Also, sufficient conditions for the existence of sharply vertex-transitive solutions to $O P\left(k^{m}\right), k m$ odd, with an additional property are provided in [8, Theorem 8.1]. The assumption that seems to be successful for constructing new symmetric solutions to the classic Oberwolfach problem is that the action of $G$ on the vertex-set is 1-rotational. The concept of a 1-rotational solution to the classic Oberwolfach problem has been formally introduced and studied, for the very first time, in [10]. In general, a $k$-factorization of a complete graph is said to be 1 -rotational under a group $G$ if it admits $G$ as an automorphism group acting sharply transitively on all but one vertex, called $\infty$, which is fixed by each element of $G$. As pointed out in [10], if a 1 -rotational $k$-factorization $\mathcal{F}$ of $K_{v}$ exists under a group $G$, then the vertices of $K_{v}$ can be renamed over $G \cup\{\infty\}$ in such a way that $G$ acts on vertices by right translation (with the condition $\infty+g=\infty$ for any $g \in G$ ) and $\mathcal{F}$ is preserved under the action of $G$, namely $F+g \in \mathcal{F}$ for any $F \in \mathcal{F}$ and $g \in G$. Of course, the graph $F+g$ is obtained by replacing each vertex of the $k$-factor $F$, say $x$, with $x+g$, for any $g \in G$. Moreover, the $k$-factorization $\mathcal{F}$ can be obtained as the $G$-orbit of any of its $k$-factors and when $k=2$ it readily follows that all cycles in $\mathcal{F}$ passing through $\infty$ have the same length.

It is well known that for each odd order group $G$ there exists a 1 -factorization which is 1 -rotational under $G,[7]$. The same result does not hold for 1-rotational 2-factorizations: groups have even order in this case and it was proved in [10] that they must satisfy some prescribed properties. It was also proved in the same paper that each 1-rotational 2 -factorization is a solution to an Oberwolfach problem. Obviously 1-rotational solutions should be more rare, nevertheless the group structure can be a useful tool to construct them. In fact, new solutions to Oberwolfach problems were constructed in [10] by working entirely in the group. In particular for each symmetrically sequenceable group $G$, [16], of order $2 n$ a 1 -rotational solution to $O P(2 n+1)$ under $G$ can be constructed. For completeness, we recall that each solvable group with exactly one involution, except for the quaternion group $Q_{8}$, is symmetrically sequenceable, [2]. A wider class of groups realizing 1-rotational solutions to the classic Oberwolfach Problem can be found in [29]. Necessary conditions for the existence of a cyclic 1-rotational solution to $\operatorname{OP}\left(3,2 l_{1}, \ldots, 2 l_{t}\right)$, with a complete characterization when $t=1$, are given in [11]. Although the concept of a 1-rotational solution to the Oberwolfach problem has been formalized and investigated in [10], it should be pointed out that some earlier results have been achieved via the 
1-rotational approach. In $[25,24,26]$ the authors provide solutions to the Oberwolfach Problem (with a special attention to the cases with two and three parameters) which are 1-rotational under the cyclic group, even though they simply speak of cyclic solutions. Finally, 1-rotational solutions to $O P\left(3^{2 n+1}\right)$ can be found in $[12,13]$.

In this paper we introduce a product of graphs, that we call the circle product and which can be applied to obtain decompositions starting from known ones. In particular we will apply the circle product to combine known solutions of the Oberwolfach Problem and get infinitely many solutions for greater orders, in both classic and non classic cases. When the circle product is applied to 1 -rotational solutions, the new obtained solutions will be 1-rotational as well.

\section{The circle product}

Let $\Gamma_{1}$ and $\Gamma_{2}$ be undirected simple graphs without isolated vertices and let $\infty$ be a fixed element which either lies in some $V\left(\Gamma_{i}\right), i \in\{1,2\}$, or not. If $\infty \in V\left(\Gamma_{i}\right)$, we will set $\Gamma_{i}^{*}=\Gamma_{i}-\{\infty\}$. If $\infty \notin V\left(\Gamma_{i}\right)$ when speaking of $\Gamma_{i}^{*}$ we will mean the same graph $\Gamma_{i}$.

For each pair $\left(e_{r}, e_{s}\right) \in E\left(\Gamma_{1}\right) \times E\left(\Gamma_{2}\right)$, we define the product $e_{r} \circ e_{s}$ to be the graph whose vertex-set and edge-set are described below:

1. If $e_{r}=[\infty, a], e_{s}=[\infty, b]$, then

$$
\begin{aligned}
& V\left(e_{r} \circ e_{s}\right)=\{\infty,(a, b)\} \\
& E\left(e_{r} \circ e_{s}\right)=\{[\infty,(a, b)]\}
\end{aligned}
$$

2. If $e_{r}=[\infty, a], e_{s}=[c, d] \in E\left(\Gamma_{2}^{*}\right)$, then:

$$
\begin{aligned}
& V\left(e_{r} \circ e_{s}\right)=\{(a, d),(a, c)\} \\
& E\left(e_{r} \circ e_{s}\right)=\{[(a, d),(a, c)]\}
\end{aligned}
$$

3. If $e_{r}=[a, b] \in E\left(\Gamma_{1}^{*}\right), e_{s}=[\infty, c]$, then:

$$
\begin{aligned}
& V\left(e_{r} \circ e_{s}\right)=\{(a, c),(b, c)\} \\
& E\left(e_{r} \circ e_{s}\right)=\{[(a, c),(b, c)]\}
\end{aligned}
$$

4. If $e_{r}=[a, b] \in E\left(\Gamma_{1}^{*}\right), e_{s}=[c, d] \in E\left(\Gamma_{2}^{*}\right)$, then:

$$
\begin{aligned}
& V\left(e_{r} \circ e_{s}\right)=\{(a, c),(a, d),(b, c),(b, d)\} \\
& E\left(e_{r} \circ e_{s}\right)=\{[(a, c),(b, d)],[(a, d),(b, c)]\}
\end{aligned}
$$


Following the above notations, we can compose the graphs $\Gamma_{1}$ and $\Gamma_{2}$ thus obtaining a new graph which is called the circle product of $\Gamma_{1}$ and $\Gamma_{2}$.

Definition 2.1. The circle product $\Gamma_{1} \circ \Gamma_{2}$ is the graph obtained as the union of all graphs $e_{r} \circ e_{s}$ as the pair $\left(e_{r}, e_{s}\right)$ varies in $E\left(\Gamma_{1}\right) \times E\left(\Gamma_{2}\right)$.

Obviously, the product $e_{r} \circ e_{s}$ changes depending on whether $e_{r}$ or $e_{s}$ contains the vertex $\infty$ or not. Besides, if $\infty \notin V\left(\Gamma_{1}\right) \cap V\left(\Gamma_{2}\right)$ then there will not be the products defined in (1), while if $\infty \notin V\left(\Gamma_{1}\right) \cup V\left(\Gamma_{2}\right)$ then there will be only the products defined in (4). Observe also that $V\left(\Gamma_{1} \circ \Gamma_{2}\right)=V\left(\Gamma_{1}^{*}\right) \times V\left(\Gamma_{2}^{*}\right) \cup\{\infty\}$ whenever $\infty \in V\left(\Gamma_{1}\right) \cap V\left(\Gamma_{2}\right)$, while $V\left(\Gamma_{1} \circ \Gamma_{2}\right)=V\left(\Gamma_{1}^{*}\right) \times V\left(\Gamma_{2}^{*}\right)$ in all the other cases. If $\infty \notin V\left(\Gamma_{1}\right) \cup V\left(\Gamma_{2}\right)$ then $\Gamma_{1} \circ \Gamma_{2}$ coincides with the usual direct product of graphs, (see [18]).

We will employ the following specific notation to denote $\Gamma_{1} \circ \Gamma_{2}$ :

- $\Gamma_{1} \diamond \Gamma_{2}$, if $\infty \in V\left(\Gamma_{1}\right) \cap V\left(\Gamma_{2}\right)$,

- $\Gamma_{1} \triangleleft \Gamma_{2}$, if $\infty \in V\left(\Gamma_{1}\right)$ and $\infty \notin V\left(\Gamma_{2}\right)$,

- $\Gamma_{1} \triangleright \Gamma_{2}$, if $\infty \notin V\left(\Gamma_{1}\right)$ and $\infty \in V\left(\Gamma_{2}\right)$,

- $\Gamma_{1} \cdot \Gamma_{2}$, if $\infty \notin V\left(\Gamma_{1}\right) \cup V\left(\Gamma_{2}\right)$.

When it is not necessary to specify whether $\infty$ lies in some $V\left(\Gamma_{i}\right)$ or not, we will preserve the notation $\Gamma_{1} \circ \Gamma_{2}$.

Obviously, when considering a graph $\Gamma_{i}, i \in\{1,2\}$, we can always label its vertices in such a way that $\Gamma_{i}$ either contains a vertex named $\infty$ or not, moreover, different choices for the vertex named $\infty$ may give rise to different graphs as a result of the circle product. If this is the case, we will specify which vertex is labeled with $\infty$.

Finally, it is easy to check that $\Gamma_{1} \circ \Gamma_{2}$ is a simple graph in all cases.

The next proposition shows what happens when we apply the circle product to some standard graphs.

Proposition 2.2. The following statements hold:

1. $K_{v} \diamond K_{w} \cong K_{(v-1)(w-1)+1}$;

2. $K_{v} \triangleleft K_{w} \cong K_{w} \triangleright K_{v} \cong K_{\{(v-1): w\}}$;

3. $\Gamma \diamond K_{2} \cong \Gamma \triangleright K_{2} \cong K_{2} \triangleleft \Gamma \cong \Gamma$ for any simple graph $\Gamma$; in particular, $C_{n} \diamond K_{2} \cong$ $C_{n} \triangleright K_{2} \cong K_{2} \triangleleft C_{n} \cong C_{n}$;

4. $C_{n} \triangleleft K_{2} \cong C_{2 n-2}$;

5. $C_{n} \cdot K_{2} \cong K_{2} \cdot C_{n} \cong\left\{\begin{array}{c}C_{n} \sqcup C_{n} \text { if } n \text { is even } \\ C_{2 n} \text { if } n \text { is odd }\end{array}\right.$ 
Proof. The proof is an easy check. Point (1) is obvious: consider any pair of distinct vertices $x, y \in V\left(\Gamma_{1}^{*}\right) \times V\left(\Gamma_{2}^{*}\right) \cup\{\infty\}$. If $x=\infty$ and $y=(a, b)$, then $[x, y]=[\infty, a] \circ[\infty, b]$ (proceed in the same manner when $y=\infty$ ). If $x=(c, d)$ and $y=(a, b)$ with $a \neq c$ and $b \neq d$, then $[x, y]$ is an edge of $[a, c] \circ[b, d]$, if $a=c$ and $b \neq d$, then $[x, y]=[\infty, a] \circ[b, d]$, while $[x, y]=[a, c] \circ[\infty, b]$ whenever $b=d$ and $a \neq c$. Concerning point (2), we just observe that $K_{v} \triangleleft K_{w}$ is the complete equipartite graph $K_{\{(v-1): w\}}$ with $w$ parts, each containing $v-1$ elements. In particular, let $\left\{a_{1}, \ldots, a_{v-1}\right\}=V\left(K_{v}\right)-\{\infty\}$, for each $x \in V\left(K_{w}\right)$, the vertices $\left(a_{1}, x\right), \ldots,\left(a_{v-1}, x\right)$ are pairwise not adjacent in $K_{v} \triangleleft K_{w}$ and form a part of $K_{\{(v-1): w\}}$. In the same manner the vertices $\left(x, a_{1}\right), \ldots,\left(x, a_{v-1}\right)$ are pairwise not adjacent in $K_{w} \triangleright K_{v}$ and form a part of $K_{\{(v-1): w\}}$.

Now, let $\Gamma$ be a simple graph and observe that both $\Gamma \diamond[\infty, b]$ and $\Gamma \triangleright[\infty, b]$ derive from $\Gamma$ by simply replacing each vertex different from $\infty$, say $a$, with $(a, b)$. In the same manner $[\infty, b] \triangleleft \Gamma$ derives from $\Gamma$ replacing each vertex $a \in V(\Gamma)$ with $(b, a)$. Thus, point (3) follows.

Finally consider a cycle $C_{n}$. If $\infty \in V\left(C_{n}\right)$ and $C_{n}=\left(\infty, a_{2}, \ldots, a_{n}\right)$ then $C_{n} \triangleleft[a, b]$ is the $(2 n-2)$-cycle whose vertices are obtained by overlapping the pair $(a, b)$ to the sequence: $a_{2}, a_{3}, \ldots, a_{n-1}, a_{n}, a_{n}, a_{n-1}, \ldots, a_{3}, a_{2}$. More precisely: $C_{n} \triangleleft[a, b]=\left(\left(a_{2}, a\right),\left(a_{3}, b\right), \ldots\right.$, $\left.\left(a_{n}, b\right),\left(a_{n}, a\right), \ldots,\left(a_{3}, a\right),\left(a_{2}, b\right)\right)$ or $C_{n} \triangleleft[a, b]=\left(\left(a_{2}, a\right),\left(a_{3}, b\right), \ldots,\left(a_{n}, a\right),\left(a_{n}, b\right), \ldots\right.$, $\left.\left(a_{3}, a\right),\left(a_{2}, b\right)\right)$ according to whether $n$ is odd or even. Furthermore, if $\infty \notin V\left(C_{n}\right)$ and $C_{n}=\left(a_{1}, \ldots, a_{n}\right)$, we have either

$$
\begin{aligned}
C_{n} \cdot[x, y]= & \left(\left(a_{1}, x\right),\left(a_{2}, y\right), \ldots,\left(a_{n-1}, x\right),\left(a_{n}, y\right)\right) \\
& \left(\left(a_{1}, y\right),\left(a_{2}, x\right), \ldots,\left(a_{n-1}, y\right),\left(a_{n}, x\right)\right)
\end{aligned}
$$

or

$$
C_{n} \cdot[x, y]=\left(\left(a_{1}, x\right),\left(a_{2}, y\right) \ldots\left(a_{n}, x\right),\left(a_{1}, y\right),\left(a_{2}, x\right) \ldots\left(a_{n}, y\right)\right)
$$

according to whether $n$ is even or odd.

In the following propositions we point out some properties of the circle product.

Proposition 2.3. Let $\Gamma_{1}$ and $\Gamma_{2}$ be simple graphs and let $(a, b)$ be a vertex of $\Gamma_{1} \circ \Gamma_{2}$, with $a \in V\left(\Gamma_{1}^{*}\right)$ and $b \in V\left(\Gamma_{2}^{*}\right)$. It is $d_{\Gamma_{1} \circ \Gamma_{2}}((a, b))=d_{\Gamma_{1}}(a) d_{\Gamma_{2}}(b)$. Moreover, if $\infty$ is in $\Gamma_{1} \circ \Gamma_{2}$ then $d_{\Gamma_{1} \circ \Gamma_{2}}(\infty)=d_{\Gamma_{1}}(\infty) d_{\Gamma_{2}}(\infty)$.

Proof. Any edge of $\Gamma_{1} \circ \Gamma_{2}$ passing through $(a, b)$ lies in a product of edges, say $e_{1} \circ e_{2}$, where $e_{1}$ and $e_{2}$ are incident with $a$ and $b$, respectively. Since the number of these mutually edge-disjoint products is $d_{\Gamma_{1}}(a) d_{\Gamma_{2}}(b)$ and any of them provides exactly one edge passing through $(a, b)$, it follows that $d_{\Gamma_{1} \circ \Gamma_{2}}((a, b))=d_{\Gamma_{1}}(a) d_{\Gamma_{2}}(b)$.

One can proceed in the same manner to get $d_{\Gamma_{1} \circ \Gamma_{2}}(\infty)=d_{\Gamma_{1}}(\infty) d_{\Gamma_{2}}(\infty)$.

As an immediate consequence, we can state that the class of regular graphs is closed under the circle product.

Proposition 2.4. The circle product of two regular graphs of degree $k$ and $t$, respectively, is a kt-regular graph. 
Proposition 2.5. If $\mathcal{F}_{1}=\left\{\Gamma_{1}, \ldots, \Gamma_{s}\right\}$ and $\mathcal{F}_{2}=\left\{\Gamma_{1}^{\prime}, \ldots, \Gamma_{r}^{\prime}\right\}$ are decompositions of the graphs $G_{1}$ and $G_{2}$, respectively, then $\mathcal{F}_{1} \circ \mathcal{F}_{2}=\left\{\Gamma_{i} \circ \Gamma_{j}^{\prime} \mid i=1, \ldots, s, j=1, \ldots, r\right\}$ is a decomposition of the graph $G_{1} \circ G_{2}$.

Proof. Let $[x, y] \in E\left(G_{1} \circ G_{2}\right)$. If $x=\infty$ and $y=(a, b), a \in V\left(G_{1}\right) b \in V\left(G_{2}\right)$, we necessarily have $[x, y]=[\infty, a] \circ[\infty, b]$. Let $\Gamma_{i}$ (respectively $\left.\Gamma_{j}^{\prime}\right)$ be the unique graph of $\mathcal{F}_{1}\left(\right.$ resp. $\mathcal{F}_{2}$ ) which contains $[\infty, a]$ (resp. $[\infty, b]$ ), then $\Gamma_{i} \circ \Gamma_{j}^{\prime}$ is the unique graph of $\mathcal{F}_{1} \circ \mathcal{F}_{2}$ containing $[x, y]$. Proceed in the same manner if $y=\infty$. Now suppose $x \neq \infty$ and $y \neq \infty$, with $x=(a, b)$ and $y=(c, d)$. If $a \neq c$ and $b \neq d$, let $\Gamma_{i}$ (respectively $\Gamma_{j}^{\prime}$ ) be the unique graph of $\mathcal{F}_{1}\left(\right.$ resp. $\mathcal{F}_{2}$ ) which contains $[a, c]$ (resp. $[b, d]$ ), then $\Gamma_{i} \circ \Gamma_{j}^{\prime}$ is the unique graph of $\mathcal{F}_{1} \circ \mathcal{F}_{2}$ containing $[x, y]$. Finally suppose $a=c$ and $b \neq d$ and let $\Gamma_{i}$ (respectively $\Gamma_{j}^{\prime}$ ) be the unique graph of $\mathcal{F}_{1}$ (resp. $\mathcal{F}_{2}$ ) which contains $[\infty, a]($ resp. $[b, d])$, then $\Gamma_{i} \circ \Gamma_{j}^{\prime}$ is the unique graph of $\mathcal{F}_{1} \circ \mathcal{F}_{2}$ containing $[x, y]$. In the same manner proceed if $a \neq c$ and $b=d$.

\section{New solutions to the classic Oberwolfach Problem}

Our constructions are presented in Theorems 3.4, 4.1 and 4.2 and need some machinery and preliminary lemmas explained below.

Let $S=\left\{e_{1}, e_{2}, \ldots, e_{w}\right\}$ be a 1 -factor of the complete graph $K_{2 w}$ and let $F_{1}, \ldots, F_{w}$ be $w$ (not necessarily distinct or edge-disjoint) 2 -factors of the complete graph $K_{2 n+1}$.

For the constructions explained in Lemma 3.1 and in Lemma 3.2, label the vertices of $K_{2 n+1}$ in such a way that $\infty \in V\left(K_{2 n+1}\right)$. For each 2 -factor $F_{i}$ denote by $\lambda_{i}$ the length of the cycle through $\infty$ and let $L_{i}$ and $M_{i}$ be multisets of even and odd integers, respectively, so that $F_{i}$ is a $F_{i}\left(\lambda_{i}, L_{i}, M_{i}\right) 2$-factor. Then we have:

Lemma 3.1. Label the vertices of $K_{2 w}$ in such a way that $\infty \in V\left(K_{2 w}\right)$ and, without loss of generality, suppose $\infty$ to be a vertex of $e_{1}$.

The graph $T=\left(e_{1} \diamond F_{1}\right) \sqcup\left(e_{2} \triangleright F_{2}\right) \sqcup \cdots \sqcup\left(e_{w} \triangleright F_{w}\right)$ is a 2-factor of $K_{2 n(2 w-1)+1}$ of type $\left(\lambda_{1}, L_{1}, M_{1}, 2\left(\lambda_{2}-1\right), L_{2}^{2}, 2 M_{2}, \ldots, 2\left(\lambda_{w}-1\right), L_{w}^{2}, 2 M_{w}\right)$.

Proof. The graph $T$ is the disjoint union of the graphs $e_{i} \circ F_{i}, i=1, \ldots, w$, and it is a subgraph of $K_{2 w} \diamond K_{2 n+1}=K_{2 n(2 w-1)+1}$. Moreover let $e_{1}=\left[\infty, b_{1}\right]$ and $e_{i}=\left[a_{i}, b_{i}\right]$, $i=2, \ldots, w$. Recalling how the circle product is defined, we have $V\left(e_{1} \diamond F_{1}\right)=\{\infty\} \cup$ $\left\{b_{1}\right\} \times V\left(K_{2 n+1}^{*}\right)$ and $V\left(e_{i} \triangleright F_{i}\right)=\left\{a_{i}, b_{i}\right\} \times V\left(K_{2 n+1}^{*}\right), i=2, \ldots, w$. Therefore $V(T)=$ $V\left(K_{2 w} \diamond K_{2 n+1}\right)$. Also, by Proposition 2.4, each graph $e_{i} \circ F_{i}$ is 2 -regular and then $T$ is a 2-factor of $K_{2 w} \diamond K_{2 n+1}$. We can determine the type of $T$ by applying Proposition 2.2. More precisely: the cycles of $e_{1} \diamond F_{1}$ have the same length as those in $F_{1}$ (see Proposition 2.2, point 3); for each $i=2, \ldots, w$, the cycle of $F_{i}$ through $\infty$ gives rise to a cycle in $e_{i} \triangleright F_{i}$ of length $2\left(\lambda_{i}-1\right)$ (see Proposition 2.2, point 4); each other cycle of $F_{i}$ of odd length gives rise to a cycle with double length and each of even length gives two cycles of the same length (this from point 5). 
Lemma 3.2. Label the vertices of $K_{2 w}$ in such a way that $\infty \notin V\left(K_{2 w}\right)$. The graph $T=\left(e_{1} \triangleright F_{1}\right) \sqcup\left(e_{2} \triangleright F_{2}\right) \sqcup \cdots \sqcup\left(e_{w} \triangleright F_{w}\right)$ is a 2-factor of $K_{\{2 n: 2 w\}}$ of type $\left(2\left(\lambda_{1}-\right.\right.$ 1), $\left.L_{1}^{2}, 2 M_{1}, \ldots, 2\left(\lambda_{w}-1\right), L_{w}^{2}, 2 M_{w}\right)$

Proof. Proceed as in the proof of Lemma 3.1 and observe that the graph $T$ is the disjoint union of the graphs $e_{i} \triangleright F_{i}, i=1, \ldots, w$, and it is a subgraph of $K_{2 w} \triangleright K_{2 n+1}=K_{\{2 n: 2 w\}}$. Moreover let $e_{i}=\left[a_{i}, b_{i}\right], i=1, \ldots, w$. Recalling how the circle product of edges is defined, we have $V\left(e_{i} \triangleright F_{i}\right)=\left\{a_{i}, b_{i}\right\} \times V\left(K_{2 n+1}^{*}\right)$. Therefore $V(T)=V\left(K_{2 w} \triangleright K_{2 n+1}\right)$. Also, by Proposition 2.4, each graph $e_{i} \circ F_{i}$ is 2-regular and then $T$ is a 2-factor of $K_{2 w} \triangleright K_{2 n+1}$. We can determine the type of $T$ applying Proposition 2.2. More precisely: the cycle of $F_{i}$ through $\infty$ gives rise to a cycle in $e_{i} \triangleright F_{i}$ of length $2\left(\lambda_{i}-1\right)$ (apply Proposition 2.2, point 3 ) ; each other cycle of $F_{i}$ of odd length gives rise to a cycle with double length and each of even length gives two cycles of the same length (from point 5).

Now, for the construction of the following Lemma 3.3, label the vertices of $K_{2 w}$ in such a way that $\infty$ is a vertex of $K_{2 w}$ which lies in $e_{1}$ and label the vertices of $K_{2 n+1}$ in such a way that $\infty \notin V\left(K_{2 n+1}\right)$. For each 2 -factor $F_{i}, i=1, \ldots, w$, let $L_{i}$ and $M_{i}$ be multisets of even and odd integers, respectively, so that $F_{i}$ is a $F_{i}\left(L_{i}, M_{i}\right) 2$-factor. Then we have:

Lemma 3.3. The graph $T=\left(e_{1} \triangleleft F_{1}\right) \sqcup\left(e_{2} \cdot F_{2}\right) \sqcup \cdots \sqcup\left(e_{w} \cdot F_{w}\right)$ is a 2-factor of $K_{\{(2 w-1):(2 n+1)\}}$ of type $\left(L_{1}, M_{1}, L_{2}^{2}, 2 M_{2}, \ldots, L_{w}^{2}, 2 M_{w}\right)$

Proof. Observe that the graph $T$ is the disjoint union of the graphs $e_{1} \triangleleft F_{1}$ and $e_{i} \cdot F_{i}, i=$ $2, \ldots, w$, and it is a subgraph of $K_{2 w} \triangleleft K_{2 n+1}=K_{\{(2 w-1):(2 n+1)\}}$. Moreover let $e_{1}=\left[\infty, b_{1}\right]$ and $e_{i}=\left[a_{i}, b_{i}\right], i=2, \ldots, w$.

Applying the rules of the circle product, we have $V\left(e_{1} \triangleleft F_{1}\right)=\left\{b_{1}\right\} \times V\left(K_{2 n+1}\right)$ and $V\left(e_{i} \cdot F_{i}\right)=\left\{a_{i}, b_{i}\right\} \times V\left(K_{2 n+1}\right)$. Therefore $V(T)=V\left(K_{2 w} \triangleleft K_{2 n+1}\right)=K_{\{(2 w-1):(2 n+1)\}}$. Also, by Proposition 2.4, each graph $e_{i} \circ F_{i}$ is 2-regular and then $T$ is a 2-factor of $K_{\{(2 w-1):(2 n+1)\}}$. As in the previous lemmas, we can determine the type of $T$ in view of Proposition 2.2: the cycles in $e_{1} \triangleleft F_{1}$ are copies of those in $F_{1}$, furthermore, if $i \in\{2, \ldots, w\}$, each cycle of $F_{i}$ of odd length gives rise to a cycle with double length and each of even length gives two cycles of the same length.

Theorem 3.4. Let $w$ be an integer and let $\mathcal{F}_{1}, \ldots, \mathcal{F}_{w}$ be $w$ (not necessarily distinct) solutions to an Oberwolfach problem of order $2 n+1$. More precisely, let $\mathcal{F}_{1}$ be a solution to $O P\left(l_{1}, \ldots, l_{t}\right)$ and for each $i=2, \ldots, w$ suppose the existence of a vertex in $K_{2 n+1}$ such that all cycles of $\mathcal{F}_{i}$ passing through it have the same length $\lambda_{i}$. For $i=2, \ldots, w$, denote by $L_{i}$ and $M_{i}$ multisets of even and odd integers, respectively, in such a way that $\mathcal{F}_{i}$ is a solution to $\mathrm{OP}\left(\lambda_{i}, L_{i}, M_{i}\right)$. Then, there exists a solution to

$$
O P\left(l_{1}, \ldots, l_{t}, 2\left(\lambda_{2}-1\right), L_{2}^{2}, 2 M_{2}, \ldots, 2\left(\lambda_{w}-1\right), L_{w}^{2}, 2 M_{w}\right)
$$

Proof. Label as $\infty$ the vertex of $K_{2 n+1}$ with the property that for each $i=2, \ldots, w$ all cycles of $\mathcal{F}_{i}$ passing through $\infty$ have length $\lambda_{i}$, Let $\left\{F_{i}^{1}, \ldots, F_{i}^{n}\right\}$ be the ordered set of 2 -factors in $\mathcal{F}_{i}$. Let $\mathcal{S}$ be a 1 -factorization of $K_{2 w}$ and denote by $S_{j}, j=1, \ldots, 2 w-$ 1 , the 1 -factors of $\mathcal{S}$. Label with $\infty$ a vertex of $K_{2 w}$ and label the edges of each $S_{j}$ 
as $E\left(S_{j}\right)=\left\{e_{1 j}, \ldots, e_{w j}\right\}$ in such a way that each edge $e_{1 j}$ contains $\infty$, for each $j=$ $1, \ldots, 2 w-1$. Now fix $r \in\{1, \ldots, n\}$ and take the 2 -factors $F_{1}^{r}, \ldots, F_{w}^{r}$, where, following the previous notation, the 2 -factor $F_{i}^{r}$ is the $r$-th factor of the 2 -factorization $\mathcal{F}_{i}$. Fix $j \in\{1, \ldots, 2 w-1\}$ and take the 1 -factor $S_{j} \in \mathcal{S}$. Now apply Lemma 3.1 and observe that the graph $T_{j r}=\left(e_{1 j} \diamond F_{1}^{r}\right) \sqcup\left(e_{2 j} \triangleright F_{2}^{r}\right) \sqcup \cdots \sqcup\left(e_{w j} \triangleright F_{w}^{r}\right)$ is a 2-factor of $K_{2 n(2 w-1)+1}$ of type $\left(l_{1}, \ldots, l_{t}, 2\left(\lambda_{2}-1\right), L_{2}^{2}, 2 M_{2}, \ldots, 2\left(\lambda_{w}-1\right), L_{w}^{2}, 2 M_{w}\right)$. To be more precise, observe that $e_{1 j} \diamond F_{1}^{r} \cong F_{1}^{r}$ from point 3 of Proposition 2.2. Therefore $e_{1 j} \diamond F_{1}^{r}$ gives rise to a set of cycles of length $l_{1}, \ldots, l_{t}$ respectively, independently from the cycle of $F_{1}^{r}$ on which $\infty$ lies.

The set $\mathcal{T}=\left\{T_{j r} \mid j=1, \ldots, 2 w-1, r=1, \ldots, n\right\}$ contains $n(2 w-1) 2$-factors of $K_{2 w} \diamond K_{2 n+1}=K_{2 n(2 w-1)+1}$. To prove that it is a 2 -factorization it is sufficient to see that each edge $[x, y] \in E\left(K_{2 w} \diamond K_{2 n+1}\right)$ appears in exactly one $T_{j r}$. Suppose $x=\infty$ and $y=(a, b)$ and then necessarily $[x, y]=[\infty, a] \circ[\infty, b]$. Let $S_{j}$ be the unique 1 -factor of $\mathcal{S}$ containing $[\infty, a]=e_{1 j}$ and let $F_{1}^{r}$ be the unique 2 -factor of $\mathcal{F}_{1}$ containing $[\infty, b]$. By construction, the 2 -factor $T_{j r}$ is the unique one containing $[x, y]$. In the same manner proceed whenever $y=\infty$. Now suppose $x \neq \infty$ and $y \neq \infty$, with $x=(a, b)$ and $y=(c, d)$. If $a \neq c$ let $S_{j}$ be the unique 1 -factor of $\mathcal{S}$ containing $[a, c]=e_{t j}(t>1)$. If $b \neq d$, respectively if $b=d$, let $F_{t}^{r}$ be the unique 2 -factor of $\mathcal{F}_{t}$ which contains $[b, d]$, respectively $[\infty, b]$. By construction, the 2 -factor $T_{j r}$ is the unique one containing $[x, y]$. Now suppose $a=c$ and $b \neq d$. Let $S_{j}$ be the unique 1 -factor of $\mathcal{S}$ containing $[\infty, a]=e_{1 j}$ and let $F_{1}^{r}$ be the unique 2 -factor of $\mathcal{F}_{1}$ containing $[b, d]$. By construction, the 2 -factor $T_{j r}$ is the unique one containing $[x, y]$.

Now suppose all $\mathcal{F}_{i}$ 's, $i=1, \ldots, w$, to be 1 -rotational under the same group $G$. It is proved in [10] that whenever $\mathcal{F}_{i}$ is 1 -rotational, then the vertex of $K_{2 n+1}$ which is fixed by $G$ has the property that all cycles through it have the same length. This was already requested by our assumption for each $\mathcal{F}_{i}, i=2, \ldots, w$ now this holds for $\mathcal{F}_{1}$ as well. Label by $\infty$ the vertex of $\mathcal{F}_{i}$ which is fixed by $G$. Suppose $l_{1}$ to be the length of all cycles of $\mathcal{F}_{1}$ passing through it, while as above, $\lambda_{i}, i=2, \ldots, w$, denotes the length of all cycles of $\mathcal{F}_{i}$ through $\infty$.

It follows from the results of [10] that for any involution $j$ of $G$ there exists at least a 2 -factor in $\mathcal{F}_{i}$ which is fixed by $j$. Moreover, the 2 -factorization $\mathcal{F}_{i}$ is obtained as the orbit of this 2 -factor under the action of a right transversal of $\left\{1_{G}, j\right\}$ in $G$. Fix an involution $j \in G$ and let $T=\left\{1_{G}=t_{1}, \ldots, t_{n}\right\}$ be an ordered right transversal of $\left\{1_{G}, j\right\}$ in $G$. For each $i=1, \ldots, w$ choose $F_{i}^{1}$ to be a 2 -factor of $\mathcal{F}_{i}$ which is fixed by $j$ and let $F_{i}^{r}=F_{i}^{1}+t_{r}, r=1, \ldots, n$. Let $H$ be a group of odd order $2 w-1$. It is well known that a 1 -factorization $\mathcal{S}$ of $K_{2 w}$ which is 1 -rotational under $H$ exists. Furthermore, $H$ acts sharply transitively on the set $\mathcal{S}=\left\{S_{1}, \ldots, S_{2 w-1}\right\}$. Let $S_{1}=\left\{e_{11}, \ldots, e_{w 1}\right\}$ with $\infty$ a vertex of $e_{11}$. For each $S_{j} \in \mathcal{S}$ let $h \in H$ be the unique element of $H$ such that $S_{j}=S_{1}+h$ and set $S_{j}=\left\{e_{1 j}, \ldots, e_{w j}\right\}$ with $e_{s j}=e_{s 1}+h, s=1, \ldots, w$. With these notations we construct the 2 -factorization $\mathcal{T}=\left\{T_{j r} \mid j=1, \ldots, 2 w-1, r=1, \ldots, n\right\}$ as above. It is of type $\left(l_{1}, \ldots, l_{t}, 2\left(\lambda_{2}-1\right), L_{2}^{2}, 2 M_{2}, \ldots, 2\left(\lambda_{w}-1\right), L_{w}^{2}, 2 M_{w}\right)$ and all cycles through $\infty$ have length $l_{1}$. It is 1 -rotational under $H \times G$. In fact for each $T_{j r} \in \mathcal{T}$ and for each pair 
$(h, g) \in H \times G$ we have

$$
T_{j r}+(h, g)=\bigcup_{i=1}^{w}\left(e_{i j}+h\right) \circ\left(F_{i}^{r}+g\right)=\bigcup_{i=1}^{w}\left(e_{i j}+h\right) \circ\left(F_{i}^{1}+t_{r}+g\right)
$$

and if we let $S_{j}+h=S_{k}$ and $t_{r}+g \in\left\{j+t_{s}, t_{s}\right\}$ (i.e., $\left\{F_{1}^{r}+g, \ldots, F_{w}^{r}+g\right\}=\left\{F_{1}^{s}, \ldots, F_{w}^{s}\right\}$ ), then we have

$$
T_{j r}+(h, g)=\bigcup_{i=1}^{w} e_{i k} \circ F_{i}^{s}=T_{k s} \in \mathcal{T}
$$

We point out that a weaker form of Theorem 3.4 appeared in [10] and concerns the case where all $\mathcal{F}_{i}$ 's coincides and then have the same type. In what follows we show a simple example of how Theorem 3.4 works.

Example 3.5. Let $G=\mathbb{Z}_{6}=\{0,1,2,3,4,5\}$, let $H=\mathbb{Z}_{3}=\{0,1,2\}$ (in the usual additive notation) and let $F_{1}^{1}=\{(\infty, 0,1,5,2,4,3)\}$ and $F_{2}^{1}=\{(\infty, 0,3)$,

$(1,5,4,2)\}$ be 2 -factors of $K_{7}$, with $V\left(K_{7}\right)=G \cup\{\infty\} . F_{1}^{1}$ and $F_{2}^{1}$ are the base factors of a 1 -rotational solution to $O P(7)$ and $O P(3,4)$, respectively. Namely $\mathcal{F}_{1}=\left\{F_{1}^{1}, F_{1}^{1}+\right.$ $\left.1, F_{1}^{1}+2\right\}=\left\{F_{1}^{1}, F_{1}^{2}, F_{1}^{3}\right\}$ and $\mathcal{F}_{2}=\left\{F_{2}^{1}, F_{2}^{1}+1, F_{2}^{1}+2\right\}=\left\{F_{2}^{1}, F_{2}^{2}, F_{2}^{3}\right\}$. Consider $K_{4}$, with $V\left(K_{4}\right)=H \cup\{\infty\}$ and let $S_{1}=\{[\infty, 0],[1,2]\}$ be a base 1 -factor of a 1 -rotational 1 -factorization $\mathcal{S}=\left\{S_{1}, S_{1}+1, S_{1}+2\right\}=\left\{S_{1}, S_{2}, S_{3}\right\}$ of $K_{4}$.

We construct the following 2 -factor of $K_{19}$, with $V\left(K_{19}\right)=(H \times G) \cup\{\infty\}$ :

$$
T_{11}=\left([\infty, 0] \diamond F_{1}^{1}\right) \sqcup\left([1,2] \triangleright F_{2}^{1}\right)
$$

It consists of the 7-cycle $A^{\prime}$ and the three 4-cycles $B^{\prime}, C^{\prime}, D^{\prime}$ below:

$$
\begin{aligned}
& A^{\prime}=(\infty,(0,0),(0,1),(0,5),(0,2),(0,4),(0,3)) \\
& B^{\prime}=((1,0),(2,0),(1,3),(2,3)) \\
& C^{\prime}=((1,1),(2,5),(1,4),(2,2)) \\
& D^{\prime}=((2,1),(1,5),(2,4),(1,2))
\end{aligned}
$$

Moreover, $\mathcal{T}=\left\{T_{11}+(h, g) \mid(h, g) \in H \times G\right\}$ turns out to be a 1 -rotational solution to $O P(7,4,4,4)$.

We can repeat the construction exchanging the role of $\mathcal{F}_{1}$ and $\mathcal{F}_{2}$. In this case we have:

$R_{11}=\left([\infty, 0] \diamond F_{2}^{1}\right) \sqcup\left([1,2] \triangleright F_{1}^{1}\right)$ which consists of a 3 -cycle $A^{\prime \prime}$, a 4 -cycle $B^{\prime \prime}$, and a $12-$ cycle $C^{\prime \prime}$, namely:

$$
\begin{aligned}
A^{\prime \prime}= & (\infty,(0,0),(0,3)) \\
B^{\prime \prime}= & ((0,1),(0,5),(0,4),(0,2)) \\
C^{\prime \prime}= & ((1,0),(2,1),(1,5),(2,2),(1,4),(2,3), \\
& \quad(1,3),(2,4),(1,2),(2,5),(1,1),(2,0)) .
\end{aligned}
$$


Moreover, $\mathcal{R}=\left\{R_{11}+(h, g) \mid(h, g) \in H \times G\right\}$ turns out to be a 1 -rotational solution to $O P(3,4,12)$.

If we identify $\mathbb{Z}_{6}$ with $\mathbb{Z}_{3} \times \mathbb{Z}_{2}$, and consider two copies of a 1 -rotational solution to $O P(3)$ under $\mathbb{Z}_{2}$, whose unique 2 -factor is $(\infty, 0,1)$, we can also identify $F_{2}^{1}$ with the 2 -factor $F=([\infty, 0] \diamond(\infty, 0,1)) \sqcup([1,2] \triangleright(\infty, 0,1))$. Therefore, $\mathcal{T}$ and $\mathcal{R}$ can be reasonably considered as the result of the recursive application of Theorem 3.4 to a solution of OP(3) and $O P(7)$.

If we take $w$ solutions $\mathcal{F}_{1}, \mathcal{F}_{2}, \ldots, \mathcal{F}_{w}$ to the Oberwolfach Problem of a fixed order $2 n+1$ with the property that for every $i=1, \ldots, n$ there exists a vertex in $K_{2 n+1}$ such that all cycles of $\mathcal{F}_{i}$ passing through it have the same length $\lambda_{i}$, then we can apply Theorem 3.4 and solve

$$
\begin{gathered}
O P\left(\lambda_{\sigma(1)}, L_{\sigma(1)}, M_{\sigma(1)}, 2\left(\lambda_{\sigma(2)}-1\right), L_{\sigma(2)}^{2}, 2 M_{\sigma(2)},\right. \\
\vdots \\
\left.2\left(\lambda_{\sigma(w)}-1\right), L_{\sigma(w)}^{2}, 2 M_{\sigma(w)}\right)
\end{gathered}
$$

where $\sigma$ denotes a permutation of $\{1,2, \ldots, w\}$. Therefore, in the case where all $\mathcal{F}_{i}$ 's are mutually distinct we can solve $w$ distinct instances of the Oberwolfach Problem as $\sigma(1)$ varies in $\{1,2, \ldots, w\}$. Also the constraint consisting of composing solutions to the Oberwolfach Problem of a fixed order can be overcome making a recursive use of Theorem 3.4 and infinite families of new solutions can be obtained. In the following Corollaries we point out just few examples, nevertheless many other combinations and recursive constructions are possible.

Corollary 3.6. If there exists a solution to $O P\left(l_{1}, \ldots, l_{t}\right)$, with $2 n+1=\sum_{1}^{t} l_{i}$, then for any positive integer $w$ there exists a solution to

$$
O P\left(l_{1}, \ldots, l_{t},(4 n)^{w}\right)
$$

Moreover, if there exists a 1-rotational solution to $O P\left(l_{1}, \ldots, l_{t}\right)$ under a symmetrically sequenceable group $G$, then (3.2) admits a 1-rotational solution under $H \times G$, for any group $H$ of order $2 w+1$.

Proof. Let $\mathcal{F}_{1}$ be a solution to $O P\left(l_{1}, \ldots, l_{t}\right)$ and let $\mathcal{F}_{2}, \ldots, \mathcal{F}_{w+1}$ be $w$ copies of a solution to $O P(2 n+1)$. By applying Theorem 3.4 we get a solution to $(3.2)$.

Now assume that $\mathcal{F}_{1}$ is 1 -rotational under a symmetrically sequenceable group $G$ and recall that $\mathcal{F}_{2}, \ldots, \mathcal{F}_{w+1}$ can be chosen to be 1 -rotational under $G$, as well. In view of the second part of Theorem 3.4, we get a 1-rotational solution to (3.2).

Therefore, if we take a symmetrically sequenceable group $G$ of order $2 n$ and consider $w+1$ copies of a 1 -rotational solution to $O P(2 n+1)$ under $G$, then a 1 -rotational solution to $O P\left(2 n+1,(4 n)^{w}\right)$ under $H \times G$ exists for each group $H$ of odd order $2 w+1$. For example, starting from the 1 -rotational solution to $O P(5,12)$ under the generalized quaternion group $Q_{16}$ presented in [10], we are able to construct a 1 -rotational solution to $O P\left(5,12,32^{w}\right)$ under $H \times Q_{16}$ for each group $H$ of order $2 w+1$.

Another application of Corollary 3.6 gives the following: 
Corollary 3.7. Let $d_{1}, d_{2}, \ldots, d_{u}$ be odd positive integers.

There exists a 1-rotational solution to

$$
O P\left(2 n+1,(4 n)^{\left(d_{1}-1\right) / 2},\left(4 n d_{1}\right)^{\left(d_{2}-1\right) / 2}, \ldots,\left(4 n d_{1} \ldots d_{u-1}\right)^{\left(d_{u}-1\right) / 2}\right)
$$

In particular, if $d_{1}=d_{2}=\ldots=d_{u}=3$ then

$$
O P\left(2 n+1,4 n, \ldots, 3^{i} 4 n, \ldots, 3^{u-1} 4 n\right)
$$

has a 1-rotational solution.

Proof. We proceed by induction on $u$ using Corollary 3.6. If $u=1$, then the existence of a 1 -rotational solution to (3.3) is a consequence of applying Corollary 3.6 starting from a 1 -rotational solution to $O P(2 n+1)$ under a symmetrically sequenceable group $G$ of order $2 n$ and setting $w=\left(d_{1}-1\right) / 2$. Now, let $u>1$, by the inductive hypothesis there exists a 1 -rotational solution $\mathcal{F}$ to

$$
O P\left(2 n+1,(4 n)^{\left(d_{1}-1\right) / 2},\left(4 n d_{1}\right)^{\left(d_{2}-1\right) / 2}, \ldots,\left(4 n d_{1} \ldots d_{u-2}\right)^{\left(d_{u-1}-1\right) / 2}\right)
$$

under $G \times H_{1} \times \cdots \times H_{u-1}$, with groups $H_{i}$ of order $d_{i}, i=1, \ldots, u-1$. Applying again Corollary 3.6 to $\mathcal{F}$ we get a 1 -rotational solution to (3.3) under $G \times H_{1} \times \cdots \times H_{u}$, with groups $H_{i}$ of order $d_{i}, i=1, \ldots, u$.

For example, the previous corollary ensures the existence of 1 -rotational solutions to $O P(2 n+1,4 n), O P(2 n+1,4 n, 12 n), O P(2 n+1,4 n, 12 n, 36 n), \ldots, O P(2 n+1,4 n, 12 n, 12 n$, $60 n), \ldots, O P(2 n+1,4 n, 4 n, 20 n), \ldots, O P(2 n+1,4 n, 4 n, 4 n, 28 n) \ldots$ and so on.

It is worth pointing out that the benefit we get from constructing a 1-rotational solution $\mathcal{F}$ to $\operatorname{OP}\left(\lambda, l_{1}, \ldots, l_{t}\right)$ under the action of some group $G$, is that a solution to $O P\left(2:(n+1) ; \lambda+1, l_{1}, \ldots, l_{t}\right)$ for the complete equipartite graph $K_{\{2:(n+1)\}}$ can be constructed as well, where $2 n+1=\lambda+\sum_{i=1}^{t} l_{i}$ and $\lambda$ denotes the length of the cycles of $\mathcal{F}$ through the vertex $\infty$ which is fixed by $G$. In fact, given a 2 -factor $F$ of $\mathcal{F}$ and denoted by $\left(\infty, a_{1}, \ldots, a_{2 u}\right)$ the cycle of $F$ through $\infty$, it suffices to construct the 2 -factor $F^{\prime}$ from $F$ as follows: delete the edge $\left[a_{u}, a_{u+1}\right]$ and add the edges $\left[\infty^{\prime}, a_{u}\right],\left[\infty^{\prime}, a_{u+1}\right]$, where $\infty^{\prime}$ is a vertex not belonging to $G \cup\{\infty\}$. The set of all $F^{\prime}$, where $F$ varies in $\mathcal{F}$, turns out to be a solution of $O P\left(2:(n+1) ; \lambda+1, l_{1}, \ldots, l_{t}\right)$. We will further deal with the equipartite variant to the Oberwolfach Problem in the next section.

Here is another application of Theorem 3.4.

Corollary 3.8. For every quadruple of non negative integers $m, r, w_{1}, w_{2}$ with both $m$ and $r$ odd and $m \geq 3$, there exists a solution to

$$
\begin{aligned}
& \text { 1. } O P\left(r m,(2 r m-2)^{w_{1}},(2 m-2)^{w_{2}},(2 m)^{w_{2}(r-1)}\right) \text {; } \\
& \text { 2. } O P\left(m^{r},(2 r m-2)^{w_{1}},(2 m-2)^{w_{2}},(2 m)^{w_{2}(r-1)}\right) \text {. }
\end{aligned}
$$

Proof. First denote by $\mathcal{F}^{\prime}$ and $\mathcal{F}^{\prime \prime}$ a solution of $O P(\mathrm{rm})$ and $O P\left(\mathrm{~m}^{r}\right)$, respectively. Now let $\mathcal{F}_{2}, \ldots, \mathcal{F}_{w_{1}+1}$ be $w_{1}$ copies of $\mathcal{F}^{\prime}$ and let $\mathcal{F}_{w_{1}+2}, \ldots, \mathcal{F}_{w_{1}+w_{2}+1}$ be $w_{2}$ copies of $\mathcal{F}^{\prime \prime}$. Also let $\mathcal{F}_{1}$ be either $\mathcal{F}^{\prime}$ or $\mathcal{F}^{\prime \prime}$. By applying Theorem 3.4 to $\mathcal{F}_{1}, \mathcal{F}_{2}, \ldots, \mathcal{F}_{w_{1}+w_{2}+1}$ we get a solution to either (1) or (2) according to whether $\mathcal{F}_{1}=\mathcal{F}^{\prime}$ or $\mathcal{F}_{1}=\mathcal{F}^{\prime \prime}$. 
We point out that applications of Theorem 3.4 provide solutions to the Oberwolfach Problem whose cycles of even length are, at least theoretically, the most. In the following theorem we use the circle product to solve new instances of the Oberwolfach Problem. In particular, this Theorem gives also solutions in which all cycles have odd length.

Theorem 3.9. Let $s$ be a positive integer and let $t_{1}, \ldots, t_{2 s}, k_{1}, \ldots, k_{2 s}$ and $d$ be positive integers such that $t_{j} \geq 3$ and $t_{j} k_{j}=3 d$, for every $j=1, \ldots, 2 s$. If each $t_{j} \neq 3$ whenever $d=2$ or 6 and if there exists a solution to $O P\left(l_{1}, \ldots, l_{r}\right)$ with $l_{1}+\cdots+l_{r}=2 d+1$, then the Oberwolfach Problem $O P\left(l_{1}, \ldots, l_{r}, t_{1}^{k_{1}}, \ldots, t_{2 s}^{k_{2 s}}\right)$ has a solution.

Proof. Let $\mathcal{D}$ be a solution to $O P\left(3^{2 s+1}\right)$ and let $\left\{D_{0}, \ldots, D_{3 s}\right\}$ be its set of 2 -factors. For each $i=0, \ldots, 3 s$, the graph $K_{d+1} \diamond D_{i}$ is a spanning subgraph of $K_{d+1} \diamond K_{6 s+3}=K_{(6 s+2) d+1}$ and $\left\{K_{d+1} \diamond D_{0}, K_{d+1} \diamond D_{1}, \ldots, K_{d+1} \diamond D_{3 s}\right\}$ turns out to be a decomposition of $K_{(6 s+2) d+1}$ into isomorphic subgraphs. Each component $K_{d+1} \diamond D_{i}$ can be decomposed into $d 2$-factors $F_{i}^{1}, \ldots, F_{i}^{d}$ of type $\left(l_{1}, \ldots, l_{r}, t_{1}^{k_{1}}, \ldots, t_{2 s}^{k_{2 s}}\right)$. In fact, let $C_{i}^{1}, \ldots, C_{i}^{2 s+1}$ be the 3 -cycles composing the 2 -factor $D_{i}$ and suppose $\infty \in V\left(C_{i}^{2 s+1}\right)$. Observe that $K_{d+1} \diamond C_{i}^{2 s+1}=$ $K_{2 d+1}$ and, using a solution to $O P\left(l_{1}, \ldots, l_{r}\right)$, we can decompose $K_{d+1} \diamond C_{i}^{2 s+1}$ into $d$ 2 -factors of type $\left(l_{1}, \ldots, l_{r}\right)$. Also, for each $1 \leq j \leq 2 s$, we have $K_{d+1} \triangleleft C_{i}^{j}=K_{\{d: 3\}}$ and, using a solution to $O P\left(d: 3 ; t_{j}\right)$ (whose existence is ensured by [23]), a 2-factorization of $K_{\{d: 3\}}$ into $d 2$-factors each containing $k_{j}$ cycles of length $t_{j}$, with $t_{j} k_{j}=3 d$, can be constructed. Since all graphs $K_{d+1} \triangleleft C_{i}^{j}$ and $K_{d+1} \diamond C_{i}^{2 s+1}$, with $i$ kept fixed, are vertexdisjoint, we can combine their 2 -factors to compose $d 2$-factors $F_{i}^{1}, \ldots, F_{i}^{d}$ of $K_{d+1} \diamond D_{i}$, thus obtaining the 2 -factorization $\left\{F_{i}^{1}, \ldots, F_{i}^{d}\right\}$ of $K_{d+1} \diamond D_{i}$. We conclude that the set $\left\{F_{i}^{1}, \ldots, F_{i}^{d}, \mid i=0, \ldots, 3 s\right\}$ is a solution to $O P\left(l_{1}, \ldots, l_{r}, t_{1}^{k_{1}}, \ldots, t_{2 s}^{k_{2 s}}\right)$.

The previous Theorem allows to solve many instances of the Oberwolfach problem. Recalling that $O P(2 d+1), O P\left(3^{(2 d+1) / 3}\right)$ with $d \equiv 1(\bmod 6)$ and $O P\left(3,4^{(d-1) / 2}\right)$ with $d$ odd always have a solution, we obtain the following:

Corollary 3.10. Let $s$ be a positive even integer and let $t_{1}, \ldots, t_{2 s}, k_{1}, \ldots, k_{2 s}$ be positive integers such that $t_{j} \geq 3$ and $t_{j} k_{j}=3 d$, for every $j=1, \ldots, 2 s$. If each $t_{j} \neq 3$ whenever $d=2$ or 6 , then there exists a solution to the following instances of the Oberwolfach Problem:

1. $O P\left(2 d+1, t_{1}^{k_{1}}, \ldots, t_{2 s}^{k_{2 s}}\right)$;

2. $O P\left(3^{(2 d+1) / 3}, t_{1}^{k_{1}}, \ldots, t_{2 s}^{k_{2 s}}\right)$, with $d \equiv 1(\bmod 6)$;

3. $O P\left(3,4^{(d-1) / 2}, t_{1}^{k_{1}}, \ldots, t_{2 s}^{k_{2 s}}\right)$, with $d$ odd.

Many other instances of the Oberwolfach problem can be solved. For example starting from the known solutions presented in [5]. The previous corollaries just give a few of them. Nevertheless not all possible instances can be obtained. For example the problem $O P(3,3,3,10)$ cannot be solved using the previous Theorem 3.9. In fact starting from the known solutions to $O P(3), O P(3,3,3), O P(3,10)$, a recursive use of Theorem 3.9 does not lead to a solution of $O P(3,3,3,10)$. 


\section{New solutions to the equipartite Oberwolfach problem}

Some variations in the proof of Theorem 3.4 leads to the following results on the equipartite Oberwolfach Problem. Cause the evident similarities, we will be more concise in the proof.

Theorem 4.1. Let $w$ be an integer and let $\mathcal{F}_{1}, \ldots, \mathcal{F}_{w}$ be $w$ (not necessarily distinct) solutions to an Oberwolfach problem of order $2 n+1$. For each $i=1, \ldots, w$ suppose the existence of a vertex in $K_{2 n+1}$ such that all cycles of $\mathcal{F}_{i}$ passing through it have the same length $\lambda_{i}$. Denote by $L_{i}$ and $M_{i}$ multisets of even and odd integers, respectively, in such a way that $\mathcal{F}_{i}$ is a solution to $\operatorname{OP}\left(\lambda_{i}, L_{i}, M_{i}\right)$. Then, there exists a solution to

$$
O P\left(2 n: 2 w ; 2\left(\lambda_{1}-1\right), L_{1}^{2}, 2 M_{1}, \ldots, 2\left(\lambda_{w}-1\right), L_{w}^{2}, 2 M_{w}\right)
$$

Proof. Without loss of generality, label as $\infty$ the vertex of $K_{2 n+1}$ with the property that each cycle of $\mathcal{F}_{i}$ passing through it has length $\lambda_{i}$, for each $i=1, \ldots, w$. Let $\left\{F_{i}^{1}, \ldots, F_{i}^{n}\right\}$ be the ordered set of 2 -factors in $\mathcal{F}_{i}$. Let $\mathcal{S}$ be a 1 -factorization of $K_{2 w}$, with $\infty \notin$ $V\left(K_{2 w}\right)$, and denote as $S_{j}, j=1, \ldots, 2 w-1$, the 1 -factors of $\mathcal{S}$. Label the edges of each $S_{j}$ as $\left\{e_{1 j}, \ldots, e_{w j}\right\}$. Fix $r \in\{1, \ldots, n\}$ and take the 2 -factors $F_{1}^{r}, \ldots, F_{w}^{r}$, where, following the previous notation, the 2 -factor $F_{i}^{r}$ is the $r$-th factor of the 2-factorization $\mathcal{F}_{i}$. Fix $j \in\{1, \ldots, 2 w-1\}$ and take the 1 -factor $S_{j} \in \mathcal{S}$. Now apply Lemma 3.2 and observe that the graph $T_{j r}=\left(e_{1 j} \triangleright F_{1}^{r}\right) \sqcup\left(e_{2 j} \triangleright F_{2}^{r}\right) \sqcup \cdots \sqcup\left(e_{w j} \triangleright F_{w}^{r}\right)$ is a 2-factor of $K_{\{2 n: 2 w\}}$ of type

$$
\left(2\left(\lambda_{1}-1\right), L_{1}^{2}, 2 M_{1}, \ldots, 2\left(\lambda_{w}-1\right), L_{w}^{2}, 2 M_{w}\right)
$$

The set $\mathcal{T}=\left\{T_{j r} \mid j=1, \ldots, 2 w-1, r=1, \ldots, n\right\}$ contains $n(2 w-1) 2$-factors of $K_{2 w} \triangleright K_{2 n+1}=K_{\{2 n: 2 w\}}$ and it is a 2-factorization of $K_{\{2 n: 2 w\}}$.

Theorem 4.2. Let $w$ be an integer and let $\mathcal{F}_{1}, \ldots, \mathcal{F}_{w}$ be $w$ (not necessarily distinct) solutions to an Oberwolfach problem of order $2 n+1$. Denote by $L_{i}$ and $M_{i}$ multisets of even and odd integers, respectively, in such a way that $\mathcal{F}_{i}$ is a solution to $\operatorname{OP}\left(L_{i}, M_{i}\right)$. Then, there exists a solution to

$$
\left.O P((2 w-1):(2 n+1)) ; L_{1}, M_{1}, L_{2}^{2}, 2 M_{2}, \ldots, L_{w}^{2}, 2 M_{w}\right)
$$

Proof. Let $\mathcal{S}$ be a 1 -factorization of $K_{2 w}$, denote by $S_{j}, j=1, \ldots, 2 w-1$, the 1 -factors of $\mathcal{S}$ and label the edges of each $S_{j}$ as $\left\{e_{1 j}, \ldots, e_{w j}\right\}$. Without loss of generality, label with $\infty$ a vertex of $K_{2 w}$ in such a way that it is a vertex of $e_{1 j}$, for each $j=1, \ldots, 2 w-1$. Label the vertices of $V\left(K_{2 n+1}\right)$ in such a way that $\infty \notin V\left(K_{2 n+1}\right)$, let $\mathcal{F}_{i}, i=1, \ldots, w$, be a solution of $O P\left(L_{i}, M_{i}\right)$ and let $\left\{F_{i}^{1}, \ldots, F_{i}^{n}\right\}$ be the ordered set of its 2 -factors. Fix $r \in\{1, \ldots, n\}$ and take the 2 -factors $F_{1}^{r}, \ldots, F_{w}^{r}$, where, following the previous notation, the 2 -factor $F_{i}^{r}$ is the $r$-th factor of the 2 -factorization $\mathcal{F}_{i}$. Fix $j \in\{1, \ldots, 2 w-1\}$ and take the 1 -factor $S_{j} \in \mathcal{S}$. Now apply Lemma 3.3 and observe that the graph $T_{j r}=\left(e_{1 j} \triangleleft F_{1}^{r}\right) \sqcup\left(e_{2 j} \cdot F_{2}^{r}\right) \sqcup \cdots \sqcup\left(e_{w j} \cdot F_{w}^{r}\right)$ is a 2-factor of $K_{(2 w-1):(2 n+1)}$ of type

$$
\left(L_{1}, M_{1}, L_{2}^{2}, 2 M_{2}, \ldots, L_{w}^{2}, 2 M_{w}\right)
$$


The set $\mathcal{T}=\left\{T_{j r} \mid j=1, \ldots, 2 w-1, r=1, \ldots, n\right\}$ contains $n(2 w-1) 2$-factors of $K_{2 w} \triangleleft K_{2 n+1}=K_{(2 w-1):(2 n+1)}$ and it is a 2-factorization of $K_{(2 w-1):(2 n+1)}$.

Corollary 4.3. For every quadruple of non negative integers $m, r, w_{1}, w_{2}$ with both $m$ and $r$ odd, $m \geq 3$ and $\left(w_{1}, w_{2}\right) \neq(0,0)$, there exists a solution to

$$
O P\left((r m-1): 2\left(w_{1}+w_{2}\right) ;(2 r m-2)^{w_{1}},(2 m-2)^{w_{2}},(2 m)^{w_{2}(r-1)}\right) .
$$

Proof. First denote by $\mathcal{F}^{\prime}$ and $\mathcal{F}^{\prime \prime}$ a solution of $O P(r m)$ and $O P\left(m^{r}\right)$, respectively. Now let $\mathcal{F}_{1}, \ldots, \mathcal{F}_{w_{1}}$ be $w_{1}$ copies of $\mathcal{F}^{\prime}$ and let $\mathcal{F}_{w_{1}+1}, \ldots, \mathcal{F}_{w_{1}+w_{2}}$ be $w_{2}$ copies of $\mathcal{F}^{\prime \prime}$. By applying Theorem 4.1 to $\mathcal{F}_{1}, \mathcal{F}_{2}, \ldots, \mathcal{F}_{w_{1}+w_{2}}$ we get a solution to $(4.3)$.

Corollary 4.4. For every quadruple of non negative integers $m, r, w_{1}, w_{2}$ with both $m$ and $r$ odd and $m \geq 3$, there exists a solution to

1. $O P\left(\left(2 w_{1}+2 w_{2}+1\right): r m ; r m,(2 r m)^{w_{1}},(2 m)^{w_{2} r}\right)$;

2. $O P\left(\left(2 w_{1}+2 w_{2}+1\right): r m ; m^{r},(2 r m)^{w_{1}},(2 m)^{w_{2} r}\right)$.

Proof. First denote by $\mathcal{F}^{\prime}$ and $\mathcal{F}^{\prime \prime}$ a solution of $O P(\mathrm{rm})$ and $O P\left(\mathrm{~m}^{r}\right)$, respectively. Now let $\mathcal{F}_{2}, \ldots, \mathcal{F}_{w_{1}+1}$ be $w_{1}$ copies of $\mathcal{F}^{\prime}$ and let $\mathcal{F}_{w_{1}+2}, \ldots, \mathcal{F}_{w_{1}+w_{2}+1}$ be $w_{2}$ copies of $\mathcal{F}^{\prime \prime}$. Also let $\mathcal{F}_{1}$ be either $\mathcal{F}^{\prime}$ or $\mathcal{F}^{\prime \prime}$. By applying Theorem 4.2 to $\mathcal{F}_{1}, \mathcal{F}_{2}, \ldots, \mathcal{F}_{w_{1}+w_{2}+1}$ we get a solution to either (1) or (2) according to whether $\mathcal{F}_{1}=\mathcal{F}^{\prime}$ or $\mathcal{F}_{1}=\mathcal{F}^{\prime \prime}$.

We have already mentioned that the bipartite Oberwolfach problem was completely solved by Piotrowski in [27]. Nevertheless, its proof is commonly deemed to be pretty involved meaning that it is to be hoped that a new and less involved proof will be provided. As a particular case of Theorem 4.1 we are able to easily solve a wide class of instances of the bipartite Oberwolfach Problem by combining known solutions of the classic one, as stated below.

Corollary 4.5. Let $L$ and $M$ be multisets of even and odd integers, respectively. If there exists a solution to $O P(\lambda, L, M)$ with a vertex such that all cycles of passing through it have length $\lambda$, then there exists a solution to

$$
O P\left(2 n: 2 ; 2(\lambda-1), L^{2}, 2 M\right)
$$

Proof. Apply Theorem 4.1 when $w=1$.

An analogous of Theorem 3.9 can also be proved.

Theorem 4.6. Let $n=6 s+3$, with $s$ a positive integer, and let $t_{1}, \ldots, t_{2 s+1}, k_{1}, \ldots, k_{2 s+1}$ and $d$ be positive integers such that $t_{j} \geq 3$ and $t_{j} k_{j}=3 d$, for every $j=1, \ldots, 2 s+1$. If each $t_{j} \neq 3$ whenever $d=2$ or 6 , then the Equipartite Oberwolfach Problem $O P(d$ : $\left.n ; t_{1}^{k_{1}}, \ldots, t_{2 s+1}^{k_{2 s+1}}\right)$ has a solution. 
Proof. Let $\mathcal{D}$ be a solution to $O P\left(3^{2 s+1}\right)$ and let $\left\{D_{0}, \ldots, D_{3 s}\right\}$ be its set of 2 -factors. For each $i=0, \ldots, 3 s$, the graph $K_{d+1} \triangleleft D_{i}$ is a spanning subgraph of $K_{d+1} \triangleleft K_{6 s+3}=$ $K_{\{d:(6 s+3)\}}$ and $\left\{K_{d+1} \triangleleft D_{0}, K_{d+1} \triangleleft D_{1}, \ldots, K_{d+1} \triangleleft D_{3 s}\right\}$ turns out to be a decomposition of $K_{\{d:(6 s+3)\}}$ into isomorphic subgraphs. Each component $K_{d+1} \diamond D_{i}$ can be decomposed into $d 2$-factors $F_{i}^{1}, \ldots, F_{i}^{d}$ of type $\left(t_{1}^{k_{1}}, t_{2}^{k_{2}}, \ldots, t_{2 s+1}^{k_{2 s+1}}\right)$. In fact, let $C_{i}^{1}, \ldots, C_{i}^{2 s+1}$ be the 3 -cycles composing the 2 -factor $D_{i}$, for each $1 \leq j \leq 2 s+1$, we have $K_{d+1} \triangleleft C_{i}^{j}=$ $K_{\{d: 3\}}$ and, using a solution to $O P\left(d: 3 ; t_{j}\right)$ (whose existence is ensured by [23]), a 2 -factorization of $K_{\{d: 3\}}$ into $d 2$-factors each containing $k_{j}$ cycles of length $t_{j}$, with $t_{j} k_{j}=3 d$, can be constructed. Since all the graphs $K_{d+1} \triangleleft C_{i}^{j}$, with $i$ kept fixed, are vertex-disjoint, we can combine their 2-factors to compose $d 2$-factors $F_{i}^{1}, \ldots, F_{i}^{d}$ of $K_{d+1} \triangleleft D_{i}$, thus obtaining the 2 -factorization $\left\{F_{i}^{1}, \ldots, F_{i}^{d}\right\}$ of $K_{d+1} \triangleleft D_{i}$. We conclude that the set $\left\{F_{i}^{1}, \ldots, F_{i}^{d}, \mid i=0, \ldots, 3 s\right\}$ is a solution to $O P\left(d:(6 s+3) ; t_{1}^{k_{1}}, \ldots, t_{2 s+1}^{k_{2 s+1}}\right)$.

\section{References}

[1] B. Alspach, P.J. Schellenberg, D.R. Stinson, D. Wagner, The Oberwolfach problem and factors of uniform odd length cycles, J. Combin. Theory Ser. A 52 (1989) 20-43.

[2] B. Anderson, E.C. Ihrig, Every finite solvable group with a unique element of order two, except the quaternion group, has a symmetric sequencing, J. Combin. Des. 1 (1993) 3-14.

[3] A. Bonisoli, M. Buratti and G. Mazzuoccolo, Doubly transitive 2-factorizations, J. Combin. Des. 15 (2006) 120-132.

[4] D. Bryant, P. Danziger, On bipartite 2-factorizations of $K_{n}-I$ and the Oberwolfach problem, J. Graph Theory, DOI: 10.1002/jgt.20538.

[5] D. Bryant and C. Rodger, Cycle decompositions, in CRC Handbook of Combinatorial Designs (C.J. Colbourn and J.H. Dinitz, Eds), CRC Press, Boca Raton, FL, 2007, pp. 373-382.

[6] D. Bryant, V. Scharaschkin, Complete solutions to the Oberwolfach problem for an infinite set of orders, J. Combin. Theory Ser. B 99 (2009) 904-918.

[7] M. Buratti, A description of any regular or 1-rotational design by difference methods, Booklet of the abstracts of Combinatorics 2000, pp. 35-52, http://www.mat. uniroma1.it/ combinat/gaeta/Combinatorics2000.pdf.

[8] M. Buratti, F. Rania, F. Zuanni, Some constructions for cyclic perfect cycle systems, Discrete Math. 299 (2005) 33-48.

[9] M. Buratti, G. Rinaldi, On sharply vertex-transitive 2-factorizations of the complete graph, J. Combin. Theory Ser. A 111 (2005) 245-256.

[10] M. Buratti, G. Rinaldi, 1-rotational $k$-factorizations of the complete graph and new solutions to the Oberwolfach problem, J. Combin. Des. 16 (2008), no. 2, 87-100.

[11] M. Buratti, G. Rinaldi, A non-existence result on cyclic cycle-decompositions of the cocktail party graph, Discrete Math. 309 (2009) 4722-4726. 
[12] M. Buratti, F. Zuanni, G-invariantly resolvable Steiner 2-designs which are 1rotational over G, Bull. Belg. Math. Soc. 5 (1998) 221-235.

[13] M. Buratti, F. Zuanni, Explicit constructions for 1-rotational Kirkman triple systems, Utilitas Math. 59 (2001) 2730.

[14] C.J. Colbourn, J.H. Dinitz, Eds., Handbook of Combinatorial Designs, second edition, CRC Press, Boca Raton, 2007.

[15] A. Deza, F. Franek, W. Hua, M. Meszka, A. Rosa, Solutions to the Oberwolfach problem for orders 18 to 40, J. Combin. Math. Combin. Comput. 74 (2010) 95-102.

[16] A.B. Evans, Complete mappings and sequencings of finite groups, in CRC Handbook of Combinatorial Designs (C.J. Colbourn and J.H. Dinitz, Eds), CRC Press, Boca Raton, FL, 2007, pp. 345-352.

[17] B. Gordon, Sequences in groups with distinct partial products, Pacific J. Math. 11 (1961) 1309-1313.

[18] R.J. Gould, Products and Hamiltonian decompositions, in CRC Handbook of Graph Theory (J. L. Gross and J. Yellen, Eds), CRC Press LLC, FL 2004, pp 269-270.

[19] R.K. Guy, Unsolved combinatorial problems, in D.J.A. Welsh (Ed.), Proceedings on the Conference on Combinatorial Mathematics and its Applications, Oxford, 1967, Academic Press, New York, 1971, p.121.

[20] D.G. Hoffman, P.J. Schellenberg, The existence of $C_{k}$-factorizations of $K_{2 n}-F$, Discrete Math. 97 (1991) 243-250.

[21] C. Huang, A. Kotzig, A. Rosa, On a variation of the Oberwolfach problem, Discrete Math. 27 (1979) 261-277.

[22] J. Liu, A generalization of the Oberwolfach problem and $C_{t}$-factorizations of complete equipartite graphs, J. Combin. Des. 8 (2000) 42-49.

[23] J. Liu, The equipartite Oberwolfach problem with uniform tables, J. Combin. Theory Ser. A 101 (2003) 20-34.

[24] M.A. Ollis, Some cyclic solutions to the three table Oberwolfach problem, Electron. J. Combin. 12 (2005).

[25] M.A. Ollis, D.A. Preece, Sectionable terraces and the (generalised) Oberwolfach Problem, Discrete Math. 266 (2003) 399-416.

[26] M.A. Ollis and A.D. Sterr, From graceful labellings of paths to cyclic solutions of the Oberwolfach problem, Discrete Math. 309 (2009) 4877-4882.

[27] W.L. Piotrowski, The solution of the bipartite analogue of the Oberwolfach problem, Discrete Math. 97 (1991) 339-356.

[28] D.K. Ray-Chaudhuri, R.M. Wilson Solution of Kirkman's schoolgirl problem, in Proc. Symp. Pure Math., Amer. Math. Soc., Providence, RI, 19 (1971) 187-204.

[29] T. Traetta, Some new results on 1-rotational 2-factorizations of the complete graph, J. Combin. Des. 18 (2010) 237-247. 